\title{
ON $q$-DERANGEMENT NUMBERS
}

\author{
MICHELLE L. WACHS
}

(Communicated by Thomas Brylawski)

\begin{abstract}
We derive a $q$-analogue of the classical formula for the number of derangements of an $n$ element set. Our derivation is entirely analogous to the classical derivation, but relies on a descent set preserving bijection between the set of permutations with a given derangement part and the set of shuffles of two permutations.
\end{abstract}

A classical application of binomial inversion (more generally the principle of inclusion-exclusion) is the derivation of the formula for the number of derangements (permutations with no fixed points) of an $n$ element set:

$$
d_{n}=n ! \sum_{k=0}^{n} \frac{(-1)^{k}}{k !} \text {. }
$$

This is obtained by counting permutations according to their number of fixed points and then inverting the resulting equation.

In this note we shall derive a formula of I. Gessel [G] for $q$-counting derangements by the major index statistic in a way entirely analogous to the classical $q=1$ case. That is, we shall $q$-count permutations with $k$ fixed points and then use Gauss inversion ( $q$-binomial inversion or more generally Möbius inversion on the lattice of subspaces of a vector space) to derive the following formula for $q$-derangement numbers:

$$
d_{n}(q)=[n] ! \sum_{k=0}^{n} \frac{(-1)^{k}}{[k] !} q^{\left(\begin{array}{c}
k \\
2
\end{array}\right)} .
$$

A key step in our derivation and an interesting result in its own right is a descent-preserving bijection between the set of permutations with a given derangement part and the set of shuffles of two permutations. This bijection enables us to use a formula of A. Garsia and I. Gessel for $q$-counting shuffles.

Gessel [G] obtained the formula for $q$-derangement numbers as a corollary of an Eulerian generating function formula for counting permutations by descents, major index, and cycle structure, which is proved via a correspondence

Received by the editors February 6, 1988.

1980 Mathematics Subject Classification (1985 Revision). Primary 05A30; Secondary 05A19, 05A15, 05A05.

Research partially supported by NSF grant DMS:8503700. 
between partitions and permutations. $q$-Derangement numbers have also been interpreted combinatorially on sets of permutations bijectively associated with derangements by A. Garsia and J. Remmel [GR] using the inversion index statistic and by $J$. Désarménien $\left[D_{2}\right]$ (see $\left[D_{1}\right]$ and $[D W]$ ) using the major index and inversion index statistics.

We shall briefly review some permutation statistic notation and terminology. For each integer $n \geq 1$, let [ $n$ ] denote the polynomial $1+q+q^{2}+\cdots+q^{n-1}$ and let $[n]$ ! denote the polynomial $[n][n-1] \cdots[1]$. Also [0]! is taken to be 1 . The $q$-binomial coefficients are given by

$$
\left[\begin{array}{l}
n \\
k
\end{array}\right]=\frac{[n] !}{[k] ![n-k] !}
$$

for $0 \leq k \leq n$.

For any positive integer $n$, let $\langle n\rangle$ denote the set $\{1,2, \ldots, n\}$. We shall think of permutations in the symmetric group $\mathscr{S}_{n}$ as words with $n$ distinct letters in $\langle n\rangle$. More generally, for a set $A$ of $n$ positive integers, $\mathscr{S}_{A}$ denotes the set of permutations of $A$ or words with $n$ distinct letters in $A$. The descent set of a permutation $\sigma=\sigma_{1}, \sigma_{2}, \ldots, \sigma_{n}$ is $\operatorname{des}(\sigma)=\left\{i \in\langle n-1\rangle \mid \sigma_{i}>\sigma_{i+1}\right\}$. The major index of $\sigma$ is maj $(\sigma)=\sum_{i \in \operatorname{des}(\sigma)} i$. Let us recall MacMahon's [M] formula for maj- $q$-counting permutations in $\mathscr{S}_{n}$ :

$$
\sum_{\sigma \in \mathscr{S}_{n}} q^{\operatorname{maj}(\sigma)}=[n] ! .
$$

A letter $i \in A$ is said to be a fixed point of $\sigma \in \mathscr{S}_{A}$ if $\sigma(i)=i$. A permutation with no fixed points is called a derangement. Let $D_{n}$ denote the set of all derangements in $\mathscr{S}_{n}$. The q-derangement numbers are defined by

$$
d_{n}(q)=\sum_{\sigma \in D_{n}} q^{\operatorname{maj}(\sigma)}
$$

It will be convenient to view the empty word $\Lambda$ as a derangement and to define $D_{0}$ to be the set $\{\Lambda\}$. We also let $\operatorname{maj}(\Lambda)=0$ and $d_{0}(q)=1$.

For any permutation $\alpha \in \mathscr{S}_{A}$, where $A=\left\{a_{1}<a_{2}<\cdots<a_{k}\right\}$, define the reduction of $\alpha$ to be the permutation in $\mathscr{S}_{k}$ obtained from $\alpha$ by replacing each letter $a_{i}$ by $i, i=1,2, \ldots, k$. The derangement part of a permutation $\sigma \in \mathscr{S}_{n}$, denoted $d p(\sigma)$, is the reduction of the subword of nonfixed points of $\sigma$. For example, $d p(5,3,1,4,7,6,2)=$ reduction of $5,3,1,7,2=4,3,1,5,2$. We shall use the convention that the derangement part of the identity permutation is the empty word $\Lambda$. Note that the derangement part of a permutation is a derangement, and that conversely, any derangement in $D_{k}$ and $k$ element subset of $\langle n\rangle$ determines a permutation in $\mathscr{S}_{n}$ with $n-k$ fixed points. Hence, the number of permutations in $\mathscr{S}_{n}$ with a given derangement part in $D_{k}$ is $\left(\begin{array}{l}n \\ k\end{array}\right)$. Our goal is to $q$-count permutations with a given derangement part.

Let $\alpha \in D_{k}$. There is an obvious bijection between the set $\left\{\sigma \in \mathscr{S}_{n} \mid d p(\sigma)=\right.$ $\alpha\}$ and the set $\operatorname{Sh}(\alpha, \beta)$ of all shuffles of $\alpha$ and $\beta=k+1, k+2, \ldots, n$, 
i.e. permutations in $\mathscr{S}_{n}$ which contain $\alpha$ and $\beta$ as complementary subwords. Indeed, for each permutation $\sigma$ in the former set, replace the subword of nonfixed points of $\sigma$ by $\alpha$ and the complementary subword of fixed points by $\beta$. A very useful result of Garsia and Gessel [GG, Theorem 3.1] allows us to $q$-count the latter set. Unfortunately, since the above-mentioned bijection does not preserve the major index, it does not help us in $q$-counting the former set. However, we shall show that there is another bijection between these two sets of permutations which, in fact, preserves descent sets.

Define a letter $\sigma_{i}$ of $\sigma=\sigma_{1}, \sigma_{2}, \ldots, \sigma_{n} \in \mathscr{S}_{n}$ to be an excedant of $\sigma$ if $\sigma_{i}>i$ and a subcedant of $\sigma$ if $\sigma_{i}<i$. Let $s(\sigma)$ and $e(\sigma)$ be the number of subcedants and excedants, respectively, of $\sigma$. We now fix $n$ and let $k \leq n$. For $\sigma \in \mathscr{S}_{k}$, let $\tilde{\sigma}$ be the permutation of $k$ letters obtained from $\sigma$ by replacing its $i$ th smallest subcedant by $i, i=1,2, \ldots, s(\sigma)$, its $i$ th smallest fixed point by $s(\sigma)+i, i=1,2, \ldots, k-s(\sigma)-e(\sigma)$, and its $i$ th largest excedant by $n-i+1, i=1,2, \ldots, e(\sigma)$. Note that $\tilde{\sigma}$ depends on $n$ as well as $\sigma$. For example, if $\sigma=\overline{3} 2 \overline{65} \underline{41}$ (with subcedants underlined and excedants overlined) and $n=8$ then $\tilde{\sigma}=638721$. If $k=n$ then $\tilde{\sigma} \in \mathscr{S}_{n}$. If $\sigma$ is a derangement then $\tilde{\sigma} \in \mathscr{S}_{A}$, where $A=\{1,2, \ldots, s(\sigma)\} \cup\{n-e(\sigma)+1, n-e(\sigma)+2, \ldots, n\}$.

Lemma 1. Let $\sigma \in \mathscr{S}_{k}, k \leq n$. Then $\operatorname{des}(\sigma)=\operatorname{des}(\tilde{\sigma})$.

Proof. Suppose $\sigma=\sigma_{1}, \sigma_{2}, \ldots, \sigma_{k}$ and $\tilde{\sigma}=\tilde{\sigma}_{1}, \tilde{\sigma}_{2}, \ldots, \tilde{\sigma}_{k}$. For each $i \in$ $\langle k-1\rangle$, we shall show $i \in \operatorname{des}(\sigma)$ if and only if $i \in \operatorname{des}(\tilde{\sigma})$, by considering the nine possible designations of subcedant $(s)$, excedant $(e)$, and fixed point $(f)$ to $\sigma_{i}$ and $\sigma_{i+1}$. First note that if $\sigma_{i}$ is a subcedant of $\sigma$ then $\tilde{\sigma}_{i} \leq \sigma_{i}$ and if $\sigma_{i}$ is an excedant of $\sigma$ then $\tilde{\sigma}_{i} \geq \sigma_{i}$.

Cases 1-3. Suppose $\left(\sigma_{i}, \sigma_{i+1}\right)$ is an $(s, s),(e, e)$, or $(f, f)$ pair. It is then clear that $\sigma_{i}<\sigma_{i+1}$ if and only if $\tilde{\sigma}_{i}<\tilde{\sigma}_{i+1}$.

Case 4. Suppose $\left(\sigma_{i}, \sigma_{i+1}\right)$ is a $(s, e)$ pair. Then we have

$$
\tilde{\sigma}_{i} \leq \sigma_{i}<i<i+1<\sigma_{i+1}<\tilde{\sigma}_{i+1},
$$

which shows that $i \notin \operatorname{des}(\sigma)$ and $i \notin \operatorname{des}(\tilde{\sigma})$.

Case 5. Suppose $\left(\sigma_{i}, \sigma_{i+1}\right)$ is a $(s, f)$ pair. Now we have

$$
\sigma_{i}<i<i+1=\sigma_{i+1} \quad \text { and } \quad \tilde{\sigma}_{i} \leq s(\sigma)<\tilde{\sigma}_{i+1},
$$

which shows that $i \notin \operatorname{des}(\sigma)$ and $i \notin \operatorname{des}(\tilde{\sigma})$.

Case 6. Suppose $\left(\sigma_{i}, \sigma_{i+1}\right)$ is a $(f, s)$ pair. Then since $\sigma_{i+1}<i+1$ and $\sigma_{i}=i$, we have

$$
\sigma_{i+1}<\sigma_{i} \text { and } \tilde{\sigma}_{i+1} \leq s(\sigma)<\tilde{\sigma}_{i} .
$$

This shows that $i \in \operatorname{des}(\sigma)$ and $i \in \operatorname{des}(\tilde{\sigma})$.

Cases 7-9. The remaining three cases are that $\left(\sigma_{i}, \sigma_{i+1}\right)$ is a $(f, e),(e, s)$, or $(e, f)$ pair. These cases are handled similarly to the previous three cases and are left to the reader. 
Theorem 2. Let $\alpha \in D_{k}, k \leq n$, and $\gamma=s(\alpha)+1, s(\alpha)+2, \ldots, n-e(\alpha)$. Then the map $\varphi:\left\{\sigma \in \mathscr{S}_{n} \mid d p(\sigma)=\alpha\right\} \rightarrow \operatorname{Sh}(\tilde{\alpha}, \gamma)$ defined by $\varphi(\sigma)=\tilde{\sigma}$ is a bijection which preserves descent sets, i.e. $\operatorname{des}(\sigma)=\operatorname{des}(\varphi(\sigma))$. Consequently, for all $J \subseteq\langle n-1\rangle$,

$$
\left|\left\{\sigma \in \mathscr{S}_{n} \mid d p(\sigma)=\alpha, \operatorname{des}(\sigma)=J\right\}\right|=|\{\sigma \in \operatorname{Sh}(\tilde{\alpha}, \gamma) \mid \operatorname{des}(\sigma)=J\}| .
$$

Proof. In view of Lemma 1, we need only show that $\varphi$ is an invertible map whose image is $\operatorname{Sh}(\tilde{\alpha}, \gamma)$. First, we claim that if $d p(\sigma)=\alpha$ then $\tilde{\sigma}$ is obtained from $\sigma$ by replacing the subword of nonfixed points of $\sigma$ by $\tilde{\alpha}$ and the subword of fixed points of $\sigma$ by $\gamma$. Indeed, the subword of fixed points of $\sigma$ is replaced by the word $s(\sigma)+1, s(\sigma)+2, \ldots, n-e(\sigma)$, which is precisely $\gamma$ since $s(\sigma)=$ $s(\alpha)$ and $e(\sigma)=e(\alpha)$. Also since $\alpha$ is the reduction of the subword of nonfixed points of $\sigma$, the position of the $i$ th smallest subcedant of $\alpha$ is the same as the position of the $i$ th smallest subcedant of $\sigma$ in the subword of nonfixed points. The same is true for the $i$ th smallest excedant. Hence each subcedant and excedant of $\sigma$ is replaced by the same letter that replaces the corresponding subcedant or excedant of $\alpha$. This means that the subword of subcedants and excedants of $\sigma$ is replaced by $\tilde{\alpha}$. We may now conclude that $\tilde{\sigma} \in \operatorname{Sh}(\tilde{\alpha}, \gamma)$.

The above description of $\tilde{\sigma}$ as a shuffle of $\tilde{\alpha}$ and $\gamma$ also implies that $\varphi$ is invertible. Indeed, if we replace the $\tilde{\alpha}$ subword of any $\tau \in \operatorname{Sh}(\tilde{\alpha}, \gamma)$ by the permutation, of the subword positions, whose reduction is $\alpha$, and the letters of the $\gamma$ subword by their positions, we obtain a unique permutation $\sigma \in \mathscr{S}_{n}$ such that $d p(\sigma)=\alpha$ and $\varphi(\sigma)=\tau$.

Remark. Although a descent set preserving bijection between $\left\{\sigma \in \mathscr{S}_{n} \mid d p(\sigma)=\right.$ $\alpha\}$ and $\operatorname{Sh}(\alpha, \beta)$, where $\beta=k+1, k+2, \ldots, n$, will not be needed in the sequel, we should point out here that one can be constructed by composing the bijection $\varphi$ with a bijection between $\operatorname{Sh}(\alpha, \beta)$ and $\operatorname{Sh}(\tilde{\alpha}, \gamma)$ described in [BW, Proof of Proposition 4.1].

Corollary 3. Let $\alpha \in D_{k}$ and $k \leq n$. Then

$$
\sum_{\substack{d p(\sigma)=\alpha \\
\sigma \in \mathscr{S}_{n}}} q^{\operatorname{maj}(\sigma)}=q^{\operatorname{maj}(\alpha)}\left[\begin{array}{l}
n \\
k
\end{array}\right] .
$$

Proof. Since $\operatorname{maj}(\sigma)$ depends only on $\operatorname{des}(\sigma)$, it follows from Theorem 2 that

$$
\begin{aligned}
\sum_{d p(\sigma)=\alpha} q^{\operatorname{maj}(\sigma)} & =\sum_{\sigma \in \operatorname{Sh}(\tilde{\alpha}, \gamma)} q^{\operatorname{maj}(\sigma)} \\
& =q^{\operatorname{maj}(\tilde{\alpha})}\left[\begin{array}{l}
n \\
k
\end{array}\right],
\end{aligned}
$$

with the last step following from Garsia-Gessel [GG, Theorem 3.1]. (For a bijective alternative proof and generalization of the Garsia-Gessel result, see [BW].) By Lemma 1, $\operatorname{maj}(\tilde{\alpha})=\operatorname{maj}(\alpha)$, which completes the proof.

Theorem 4. For all $n \geq 0$,

$$
d_{n}(q)=[n] ! \sum_{k=0}^{n} \frac{(-1)^{k}}{[k] !} q^{\left(\begin{array}{c}
k \\
2
\end{array}\right)}
$$


Proof. By maj- $q$-counting the permutations in $\mathscr{S}_{n}$ according to derangement part and applying Corollary 3 , we obtain

$$
\begin{aligned}
{[n] ! } & =\sum_{\sigma \in \mathscr{S}_{n}} q^{\operatorname{maj}(\sigma)} \\
& =\sum_{k=0}^{n} \sum_{\alpha \in D_{k}} \sum_{d p(\sigma)=\alpha} q^{\operatorname{maj}(\sigma)} \\
& =\sum_{k=0}^{n} \sum_{\alpha \in D_{k}} q^{\operatorname{maj}(\alpha)}\left[\begin{array}{l}
n \\
k
\end{array}\right] \\
& =\sum_{k=0}^{n}\left[\begin{array}{l}
n \\
k
\end{array}\right] d_{k}(q) .
\end{aligned}
$$

Gauss inversion [A, p. 96] on the resulting equation yields,

$$
\begin{aligned}
d_{n}(q) & =\sum_{k=0}^{n}\left[\begin{array}{l}
n \\
k
\end{array}\right](-1)^{n-k} q^{\left(\frac{n-k}{2}\right)}[k] ! \\
& =\sum_{k=0}^{n} \frac{[n] !}{[n-k] !}(-1)^{n-k} q^{\left(\frac{n-k}{2}\right)},
\end{aligned}
$$

which is equivalent to the desired formula.

\section{ACKNOWLEDGMENT}

I am grateful to Adriano Garsia for the stimulating conversations which lead me to consider $q$-derangement numbers.

\section{REFERENCES}

[A] M. Aigner, Combinatorial theory, Springer-Verlag, New York, 1979.

[BW] A. Björner and M. Wachs, q-Hook length formulas for forests, J. Combin. Th. Ser A (to appear).

[D 1$]$ J. Désarménien, Une autre interprétation du nombre de dérangements, Actes $8^{e}$ Séminaire Lotharingien de Combinatoire, I.R.M.A. Strasbourg, 1984, pp. 11-16.

$\left[\mathrm{D}_{2}\right]$ J. Désarménien, personal communication.

[G] I. Gessel, Counting permutations by descents, greater index, and cycle structure, unpublished work.

[GG] A. M. Garsia and I. Gessel, Permutation statistics and partitions, Adv. in Math. 31 (1979), 288-305.

[GR] A. M. Garsia and J. Remmel, A combinatorial interpretation of q-derangement and q-Laguerre numbers, European J. Combin. 1 (198C), 47-59. 
[M] P. A. MacMahon, The indices of permutations and the derivation therefrom of functions of a single variable associated with permutations of any assemblage of objects, Amer. J. Math. 35 (1913), 281-322; reprinted in Percy Alexander MacMahon: Collected papers, vol. 1 (G. E. Andrews, ed.), M.I.T. Press, Cambridge MA., 1978, pp. 508-549.

[DW] J. Désarménien and M. Wachs, Descentes des dérangements et mots circulaires, Actes 19 Séminaire Lotharinjien de Combinatoire, I.R.M.A. Strasbourg, 1988, 13-21.

Department of Mathematics, University of Miami, Coral Gables, Florida 33124

Current address: Department of Mathematics, 201 Walker Hall, University of Florida, Gainsville, Florida 32611 